\title{
MEASUREMENTS OF ACID VOLATILE SULFIDE AND SIMULTANEOUSLY EXTRACTED METALS ARE IRREPRODUCIBLE AMONG LABORATORIES
}

\author{
Chad R. Hammerschmidt*† and G. Allen Burton JR. $\ddagger$ \\ $\dagger$ Department of Earth \& Environmental Sciences, Wright State University, 3640 Colonel Glenn Highway, Dayton, Ohio 45435 \\ $\ddagger$ School of Natural Resources and Environment, University of Michigan, Ann Arbor, Michigan 48109
}

(Submitted 16 November 2009; Returned for Revision 5 January 2010; Accepted 26 January 2010)

\begin{abstract}
Partitioning with solid phases is a principal control on availability and associated toxicity of metals to aquatic biota. In anoxic sediments, environmentally active fractions of sulfide and associated metals are defined operationally as acid-volatile sulfide (AVS) and simultaneously extracted metals (SEM). Ratios of these chemical parameters are often used in establishing equilibrium partitioning sediment benchmarks for toxicity and, therefore, require analytical accuracy to be useful. To investigate the reproducibility and accuracy of AVS and SEM measurements, we distributed subsamples of four physicochemically disparate stream sediments to seven independent laboratories, including our own, for analysis of both AVS and SEM (Cd, Cu, Pb, Ni, and Zn). Synthesis of these results shows that AVS varied from 70 to $3,500 \times$ and SEM ranged from 17 to $60 \times$ among laboratories for each of the four sediments. Inadequate detection limits for AVS precluded calculation of SEM:AVS ratios for two of the deposits, whereas the ratio varied more than 50-fold among laboratories for the other two sediments. This work highlights the need for improved quality control and standardization of methods for determination of AVS and SEM in sediments, and suggests that predictions of metal toxicity in sediments can be laboratory specific, which raises concerns on the use of the AVS:SEM model for risk assessments and regulatory decisions. Environ. Toxicol. Chem. 2010;29:14531456. (C) 2010 SETAC
\end{abstract}

Keywords-Analytical chemistry Aquatic toxicology Sediment assessment Metal bioavailability

\section{INTRODUCTION}

The U.S. Environmental Protection Agency (U.S. EPA) has recommended the use of acid-volatile sulfides (AVS) and their relationship to simultaneously extracted metals (SEM) as predictors of the bioavailability of some divalent metals in sediments (e.g., [1,2]). Sediment metals are likely to be non- or less toxic if the concentration of AVS is greater than that of SEM. Multiple laboratory and field studies have supported use of the AVS and SEM relationship for assessing toxicity [1,3-9]. The SEM:AVS approach also has been recommended for establishment of sediment guidelines for the European Union through the Water Framework Directive ([10-12]; www.icmm.com; [13]). Adoption of this approach by the United States and Western Europe for protecting aquatic biota from toxic metal exposures points to the importance of accurate determination of AVS and SEM in deposits.

Acid-volatile sulfide is defined operationally as the sulfide fraction that is evolved from sediment when treated with acid. It is a complex and variable fraction of sediment represented by a variety of reduced sulfur components, although often dominated by relatively labile Fe and Mn monosulfides [10]. Greater AVS concentrations are associated typically with organic-rich, anoxic deposits and lower levels are found usually in oxic sediments having low organic content [11]. While the SEM:AVS model can underestimate potential bioavailability of several metals in anoxic sediments (e.g., $\mathrm{Cu}, \mathrm{Hg}, \mathrm{Ni}$; [14]), inadvertent oxidation of sediment during sampling or analysis can decrease AVS and metal-binding capacity [15-17], thereby making accurate predictions of metal risk problematic. An

\footnotetext{
* To whom correspondence may be addressed (chad.hammerschmidt@wright.edu).

Published online 17 March 2010 in Wiley InterScience (www.interscience.wiley.com)
}

added complication with use of the SEM:AVS model is that multiple techniques, employing different chemical reagents and methodologies, have been used to extract AVS from sediment [10]. The efficiency of sulfide extraction from minerals, including refractory pyrite, varies among methods [18]. Accordingly, and while remaining an operational determination, extraction with $1 \mathrm{~N} \mathrm{HCl}$ has been proposed, in part, to promote greater intercomparability of AVS and SEM results [19].

The objective of this study was to conduct an interlaboratory comparison of AVS and SEM measurements, made with accepted protocols, to determine if analytical variability is a substantial source of uncertainty to toxicological predictions based on the AVS:SEM model. Here we show for four different sediment matrixes that levels of both AVS and SEM vary by orders of magnitude among seven independent laboratories, which raises concerns about the use of the AVS:SEM model for risk assessments and regulatory decisions.

\section{MATERIALS AND METHODS}

Sediments for the present study were sampled from four streams in southwest Ohio, USA: Little Sugar Creek, the Mad and Little Miami Rivers, and Warden Ditch. These streams were selected to provide a range of AVS and SEM concentrations that are representative of deposits in other freshwater systems [20]. Bulk surface sediments $(\approx 2 \mathrm{~L})$ were sampled from each stream following U.S. EPA-recommended guidelines [21], homogenized by rigorous stirring, and aliquots divided into $125-\mathrm{cm}^{3}$ polyethylene specimen cups that were filled completely (i.e., no head space), sealed hermetically, and frozen $\left(<-20^{\circ} \mathrm{C}\right)$. While stirring of the samples likely compromised the original AVS content of the parent sample, it was necessary to ensure relative homogeneity among aliquots distributed to each analytical laboratory. 
Frozen subsamples of sediment from each of the four streams (one cup of each) were distributed in conventional coolers with blue ice by overnight delivery to seven independent analytical laboratories; Alloway (Lima, OH, USA), Alpha Analytical (Mansfield, MA, USA), CRG Labs (Torrance, CA, USA), Pace Analytical Labs (Green Bay, WI, USA), SF Analytical Labs (New Berlin, WI, USA), TestAmerica Labs (Pittsburgh, PA, USA), and our own laboratory at Wright State University (WSU), which received two cups of each sediment type. All laboratories (identified below by alphabetical code, in no particular order) were instructed to determine AVS, SEM $(\mathrm{Cd}, \mathrm{Cu}, \mathrm{Pb}, \mathrm{Ni}$, and $\mathrm{Zn})$, and percent solids; each laboratory claimed an ability to make these measurements and most indicated that they followed the U.S. EPA protocol for AVS and SEM analysis [19]. This method uses cold $1 \mathrm{~N} \mathrm{HCl}$ to extract AVS and SEM. Contracted laboratories were not informed that results would be used for interlaboratory comparison; this was done to ensure that the same routine practices conducted by each laboratory were not overly scrutinized (i.e., business as usual). Most laboratories used inductively coupled plasma mass spectrometry (ICP-MS) for metals determination in the simultaneously extracted phase (e.g., U.S. EPA Method 6020), although the method of sulfide quantification (e.g., gravimetric, colorimetric, electrochemical) was not reported typically. At WSU, AVS/SEM extraction strictly followed the U.S. EPA protocol [19], with colorimetric sulfide analysis and metals determination by ICP-MS. Most quality control/assurance results (e.g., blanks, matrix spike recoveries) that accompanied data reports appeared to be acceptable (Table 1), the major exception being that several measurements of AVS and a few individual metals in the SEM fraction were below detection limits of particular laboratories.

\section{RESULTS AND DISCUSSION}

Method standardization is an approach to ensure comparable results are generated by experienced and nonexperienced, but laboratory-skilled, personnel for a given analyte. Standard methods often have the benefit of certified calibration standards and reference materials. Such is the case for the determination of most metals in sediments, for example, where there are multiple private/government vendors of both aqueous metals standards and matrix-specific certified reference materials, in addition to time-tested methodologies for sample preparation $[22,23]$. This has promoted analytical agreement within and among most laboratories; reproducibility is often less than $\pm 10 \%$ for many total metals analyses $[23,24]$. In contrast, extraction of AVS and SEM is operational, and although prescribed a U.S. EPA protocol [19], may be subject to greater variability within and among laboratories, especially given that that there is neither a commercial aqueous standard for sulfide nor reference material for AVS/SEM in sediment, although sulfide solutions can be prepared and standardized iodometrically by individual laboratories $[19,23]$.

We found that measurements of sediment AVS, made by reputable and admittedly knowledgeable laboratories, varied by 70 to $3500 \times$ among labs for each of the four sediments examined in this study (Fig. 1a-d). About half of the laboratories were unable to detect AVS in the two low-sulfide deposits, Little Sugar Creek and Mad River (Fig. 1a,b). The commercial laboratories reported detection limits from 0.002 to $0.5 \mu \mathrm{mol} / \mathrm{g}$ dry weight, which were comparable to, or much greater than, our own detection limit for sulfide (Table 1).

Simultaneously extracted metals also ranged widely among the seven laboratories, differing by a factor of 17 to $60 \times$ for each of the four deposits (Fig. 1e-h). As a result of the noted variability in AVS and SEM, calculated SEM:AVS was highly uncertain. Indeed, and while inadequate detection limits of two of the laboratories for AVS precluded calculation of SEM:AVS for Little Sugar Creek and Mad River sediments (Fig. 1i,j), the ratio differed by 50-fold among laboratories for Little Miami River and Warden Ditch deposits (Fig. 1k,1). Interlaboratory differences of SEM:AVS were most problematic for the Mad and Little Miami River deposits, where SEM:AVS was calculated to be both greater and less than unity, a ratio often used to predict a lack of toxicity.

The impetus for this study was to demonstrate that commercially contracted laboratories can obtain comparable results for AVS and SEM in sediment and, by extension, SEM:AVS ratios. Our initial assumption, and potentially that of other researchers, was that determination of AVS and SEM was relatively standardized and required little additional scrutiny. This interlaboratory comparison indicates that AVS, SEM, and SEM:AVS results can be laboratory-specific and, unfortunately, differ by orders of magnitude for a particular sample among laboratories.

The U.S. EPA guidance for deriving equilibrium partitioning sediment benchmarks (ESBs) for the protection of benthic organisms from metal mixtures recommends the following

Table 1. Summary of pertinent quality control results during analysis of acid-volatile sulfide (AVS) and simultaneously extracted metals (SEM) in four stream sediments by seven independent laboratories

\begin{tabular}{|c|c|c|c|c|c|c|c|c|}
\hline \multirow[b]{3}{*}{ Lab ID } & \multirow[b]{3}{*}{ Storage time $(\mathrm{d})$} & \multirow[b]{3}{*}{ AVS DL $(\mu \mathrm{mol} / \mathrm{g} \text { dry } w \mathrm{t})^{\mathrm{b}}$} & \multicolumn{4}{|c|}{ Percent recovery } & \multirow{2}{*}{\multicolumn{2}{|c|}{$\begin{array}{l}\text { Procedural repro- } \\
\text { ducibility (RPD) }^{\mathrm{a}}\end{array}$}} \\
\hline & & & \multicolumn{2}{|c|}{ Matrix spikes } & \multicolumn{2}{|c|}{$\begin{array}{l}\text { Laboratory control } \\
\text { sample }\end{array}$} & & \\
\hline & & & SEM & $\mathrm{AVS}^{\mathrm{c}}$ & SEM & AVS & SEM & AVS \\
\hline $\mathrm{WSU}^{\mathrm{d}}$ & 11 & 0.001 & - & $98 \pm 4$ & - & - & 26 & 11 \\
\hline A & 4 & 0.002 & - & $86 \pm 3$ & - & - & - & - \\
\hline B & 6 & 0.5 & 74 & 100 & 100 & 100 & - & - \\
\hline $\mathrm{C}$ & 3 & 0.04 & - & - & - & - & - & - \\
\hline $\mathrm{D}$ & 18 & 0.25 & 95 & 102 & 94 & 96 & 10 & - \\
\hline $\mathrm{E}$ & 12 & 0.003 & 87 & 104 & 143 & 93 & - & - \\
\hline $\mathrm{F}$ & 3 & 0.006 & - & - & - & - & - & - \\
\hline
\end{tabular}

${ }^{a}$ Relative percent difference between duplicate analyses. AVS in the sample replicated by Lab D was below detection limit.

${ }^{\mathrm{b}} \mathrm{DL}=$ detection limit.

${ }^{\mathrm{c}}$ Mean \pm one standard deviation.

${ }^{\mathrm{d}}$ Wright State University. 

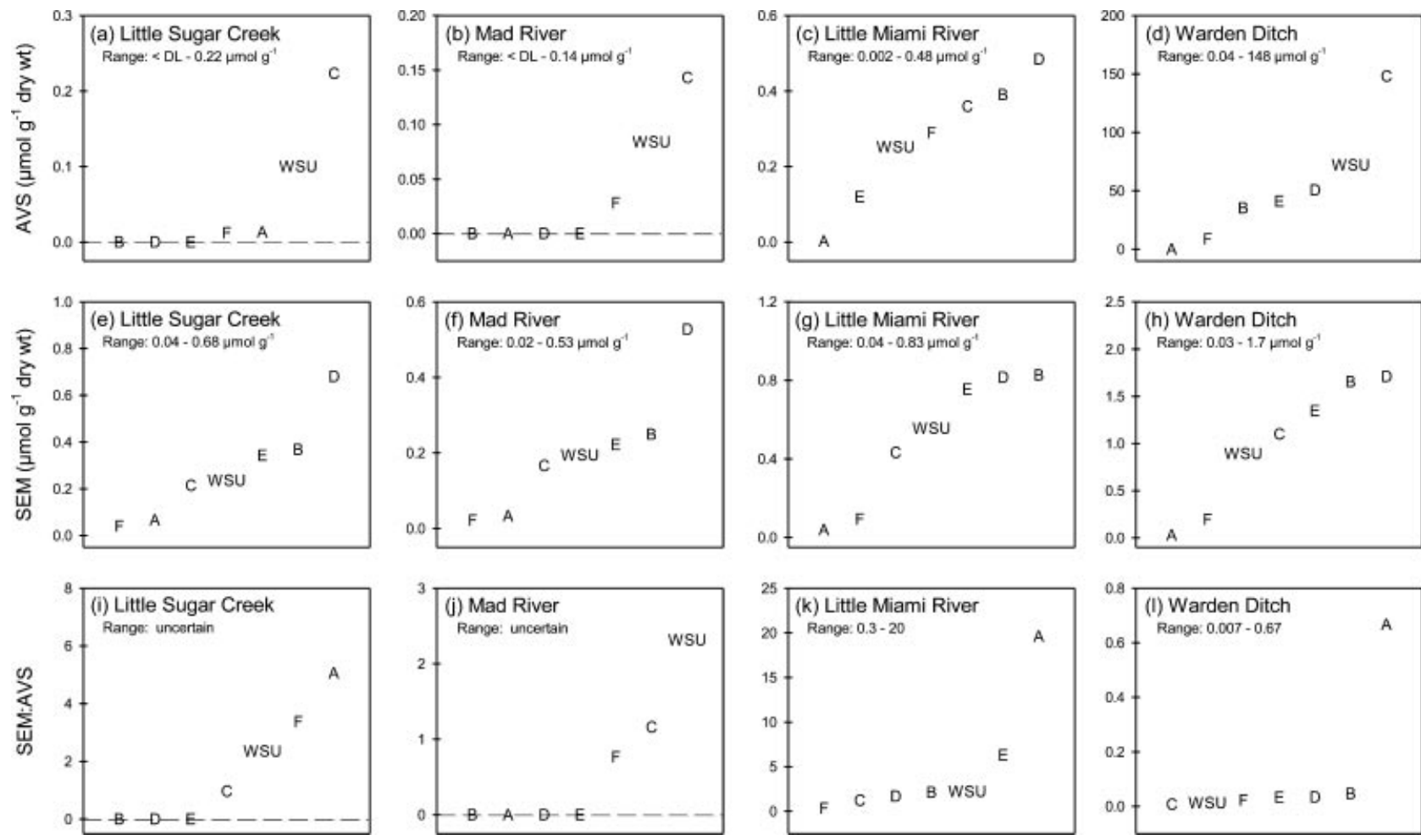

Fig. 1. Acid-volatile sulfide (AVS), simultaneously extracted metals (SEM), and SEM:AVS ratio determined by seven, independent analytical laboratories for four stream sediments in southwest Ohio, USA. Results are shown in increasing numerical value and identified by assigned laboratory identification letters. Identification letters on dashed lines denote that either AVS was below the detection limit (DL) or SEM:AVS is undefined.

methods for sulfide determination: gravimetry [1,25], colorimetry [18,26], gas chromatographic photoionization [27,28], and ion-specific electrochemistry [16,29-31]. This wide range of acceptable methods could create a potential for interlaboratory variation; however, comparison of the colorimetric and gravimetric methods, for example, found no significant difference in sediment AVS when conducted in the same laboratory [26].

Sample heterogeneity is another potential source of interlaboratory variation. Heterogeneity could result from incomplete mixing of the sediments before we divided them among specimen cups and would be apparent from inter- and intra-cup comparisons of AVS, SEM, and percent solids. In contrast to AVS and SEM, determinations of percent solids (weight:weight) were in good agreement among all laboratories across a broad range of deposit types (mean \pm standard deviation among laboratories): Little Sugar Creek $(82.3 \pm 3.5 \%)$, Mad River $(84.0 \pm 2.9 \%)$, Little Miami River (66.2 $\pm 1.5 \%)$, and Warden Ditch $(29.2 \pm 2.3 \%)$. Moreover, the WSU laboratory examined differences of AVS and SEM between two randomly selected cups of homogenized sediments from both the Little Sugar Creek and Mad River. Inter-cup variability averaged $10 \pm 4$ relative percent difference (RPD) for AVS and $15 \pm 11$ RPD for SEM among the two sediment types. These results are consistent with the degree of intra-cup variability of AVS and SEM, determined from procedurally duplicated analyses (i.e., extraction and analysis of replicate subsamples from the same cup) of deposits from Little Sugar Creek by WSU and Mad River by Lab D (Table 1). Hence, comparisons of percent solids and laboratory-specific tests of AVS and SEM suggest that variability associated potentially with heterogeneity $(<30 \%)$ is substantially less than that observed among laboratories for AVS and SEM, which was greater than $1000 \%$.
Interlaboratory variability of AVS also could result from oxidation of reduced sulfur species during transportation, storage, and analysis. Iron monosulfides, for example, oxidize rapidly when exposed to oxygen [32]. Oxidation of iron monosulfides and other labile metal sulfides would result in a lower measured AVS concentration and potentially more variable SEM level, depending on phase partitioning of the liberated metal. To minimize AVS oxidation during transportation and storage, samples were distributed to laboratories in a frozen condition - by overnight delivery - and AVS/SEM extractions were performed by each laboratory within $18 \mathrm{~d}$ of receipt (Table 1). Sediment AVS is stable for at least $56 \mathrm{~d}$ when stored either refrigerated or frozen [26]. Moreover, if sulfide oxidation during sample transport and storage were a major source of variability among laboratories, then one might expect measured AVS to be related inversely with the amount of time between receipt and analysis of samples (i.e., storage time). However, there was no significant correlation between storage time and measured AVS among the seven laboratories for each of the four sediments; Little Sugar Creek $(r=-0.34, p=0.46)$, Mad River $(r=-0.33, p=0.47)$, Little Miami River $(r=0.31$, $p=0.49)$, and Warden Ditch $(r=-0.01, p=0.98)$. These results do not unequivocally exclude oxidation during transportation and storage as a source of variability among laboratories; however, and when considered with results from the homogeneity tests, they imply that much of the variability results from laboratory-specific practices that introduce bias.

We infer that the large interlaboratory variations of AVS and SEM result from differences in the method by which they are extracted from sediments in each laboratory. Such variability results most likely from either AVS oxidation during sample preparation/analysis or operational differences in extraction. Indeed, and as noted, efficiencies of AVS extraction 
vary substantially among techniques [10]. Bias from either source cannot be evaluated entirely from standard additions, procedural replication, or even laboratory control samples if the matrix and associated lability of reduced sulfur species in the control differs substantially from that of the natural sediment. Clearly, there is a need to certify or standardize a sediment reference material for AVS and SEM from which to evaluate and assure the quality of results.

It is disconcerting that AVS and SEM values vary by orders of magnitude among laboratories, given the use of ESBs for regulatory purposes and decisions affecting remediation. The AVS method should be standardized with interlaboratory testing to better define critical methodological parameters and expected intra- and interlaboratory variances associated with these methods.

Acknowledgement-Katlin Bowman conducted metals analyses at Wright State University, and Kevin Custer and Keith Taulbee collected and distributed sediments to analytical laboratories. Support for this study was provided by the Wright State Research Challenge Program.

\section{REFERENCES}

1. DiToro DM, Mahony JD, Hansen DJ, Scott KJ, Hicks MB, Mayr SM, Edmond MS. 1990. Toxicity of cadmium in sediments: The role of acid volatile sulfide. Environ Toxicol Chem 9:1487-1502.

2. U.S. Environmental Protection Agency. 2005. Procedures for the derivation of equilibrium partitioning sediment benchmarks (ESBs) for the protection of benthic organisms: Metal mixtures (cadmium, copper, lead, nickel, silver, zinc). EPA/600/R-02/011. Office of Research and Development, Washington, DC.

3. Boothman WS, Hansen DJ, Berry WJ, Robson DL, Helmstetter A, Corbin JM, Pratt SD. 2001. Biological response to variation of acidvolatile sulphides and metals in field-exposed spiked sediments. Environ Toxicol Chem 20:264-272.

4. Griethuysen CV, von Baren J, Peeters ETHM, Koelmans AA. 2004. Trace metal availability and effects on benthic community structure in floodplain lakes. Environ Toxicol Chem 23:668-681.

5. Han J, Ma D, Quan X, Wang J, Yan Q. 2005. Bioavailability of zinc in the sediment to the estuarine amphipod Grandidierella japonica. Hydrobiologia 541:149-154.

6. Peng S-H, Wang W-X, Li X, Yen Y-F. 2004. Metal partitioning in river sediments measured by sequential extraction and biomimetic approaches. Chemosphere 57:839-851.

7. Lee J-S, Lee J-H. 2005. Influence of acid volatile sulfides and simultaneously extracted metals on the bioavailability and toxicity of a mixture of sediment-associated $\mathrm{Cd}, \mathrm{Ni}$, and $\mathrm{Zn}$ to polychaetes Neanthes arenaceodentata. Sci Total Environ 338:229-241.

8. Burton GA Jr, Nguyen LTH, Janssen C, Baudo R, McWilliam R, Bossuyt B, Beltrami M, Green A. 2005. Field verification of sediment zinc toxicity. Environ Toxicol Chem 24:541-553.

9. Burton GA Jr, Nguyen LTH, Janssen C, Baudo R, Schlekat C. 2005. Field verification of nickel sediment toxicity: Final report. Nickel Producers Environmental Research Association, Durham, NC.

10. Rickard D, Morse JW. 2005. Acid volatile sulfide (AVS). Mar Chem 97:141-197.

11. Landner L, Reuther R. 2004. Metals in Society and in the Environment: A Critical Review of Current Knowledge on Fluxes, Speciation, Bioavailability and Risk for Adverse Effects of Copper, Chromium, Nickel and Zinc. Kluwer, Dordrecht, The Netherlands.

12. International Council on Mining and Metals. 2007. MERAG: Metals Environmental Risk Assessment Guidance. London, UK.
13. U.S. Environmental Protection Agency. 2007. Framework for metals risk assessment. EPA/120/R-07/001. Office of the Science Advisor, Washington, DC.

14. Cooper DC, Morse JW. 1998. Biogeochemical controls on trace metal cycling in anoxic marine sediments. Environ Sci Technol 32:327330 .

15. Hare L, Carignan R, Huerta-Diaz MA. 1994. A field-study of metal toxicity and accumulation by benthic invertebrates - Implications for the acid-volatile sulfide (AVS) model. Limnol Oceanogr 39:1653-1668.

16. Leonard EN, Cotter AM, Ankley GT. 1996. Modified diffusion method for analysis of acid volatile sulfides and simultaneously extracted metals in freshwater sediments. Environ Toxicol Chem 15:1479-1481.

17. Zhuang Y, Allen HE, Fu G. 1994. Effect of aeration of sediment on cadmium binding. Environ Toxicol Chem 13:717-724.

18. Cornwell JC, Morse JW. 1987. The characterization of iron sulfide minerals in anoxic marine sediments. Mar Chem 22:193-206.

19. Allen HE, Fu G, Boothman W, DiToro D, Mahony JD. 1991. Draft analytical method for determination of acid volatile sulfide in sediment. U.S. Environmental Protection Agency, Washington, DC.

20. Burton GA Jr, Green A, Baudo R, Forbes V, Nguyen LTH, Janssen CR, Kukkonen J, Leppanen M, Maltby L, Soares A, Kapo K, Smith P, Dunning J. 2007. Characterizing sediment acid volatile sulfide concentrations in European streams. Environ Toxicol Chem 26:1-12.

21. U.S. Environmental Protection Agency. 2001. Methods for collection, storage, and manipulation of sediments for chemical and toxicological analyses: technical manual. EPA-823-N-01-002. Office of Water, Washington DC

22. U.S. Environmental Protection Agency. 1996. Method 3050B: Acid digestion of sediments, sludges, and soils. U.S. Environmental Protection Agency, Washington, DC.

23. American Public Health Association, American Water Works Association, and Water Environment Federation. 1995. Standard Methods for the Examination of Water and Wastewater, 19th ed. American Public Health Association, Washington, DC.

24. Coquery M, Azemard S, de Mora SJ. 2000. World-wide intercomparison exercise for the determination of trace elements and methylmercury in estuarine sediment sample IAEA-405. IAEA/AL/127. International Atomic Energy Agency, Monaco.

25. Leonard EN, Mattson VR, Benoit DA, Hoke RA, Ankley GT. 1993. Seasonal variation of acid volatile sulfide concentration in sediment cores from three northeastern Minnesota lakes. Hydrobiologia 271: 87-95.

26. Allen HE, Fu GM, Deng BL. 1993. Analysis of acid volatile sulfide and simultaneously extracted metals for the estimation of potential toxicity in aquatic sediments. Environ Toxicol Chem 12:1441-1453.

27. Casas AM, Crecelius EA. 1994. Relationship between acid volatile sulfide and the toxicity of zinc, lead and copper in marine sediments. Environ Toxicol Chem 13:529-536.

28. Slotton DG, Reuter JE. 1994. Heavy metals in intact and resuspended sediments of a California reservoir, with emphasis on potential bioavailability of copper and zinc. Mar Freshw Res 46:257-265.

29. Boothman WS, Helmstetter A. 1992. Vertical and seasonal variability of acid volatile sulfides in marine sediments. U.S. Environmenta Protection Agency, Narragansett, RI

30. Brouwer H, Murphy TP. 1994. Diffusion method for the determination of acid-volatile sulfides (AVS) in sediment. Environ Toxicol Chem $13: 1273-1275$

31. Brumbaugh WG, Ingersoll CG, Kemble NE, May TW, Zajcek JL. 1994 Chemical characteristics of sediments and interstitial water from the upper Clark Fork River and Milltown Reservoir, Montana. Environ Toxicol Chem 13:1971-1983.

32. Burton ED, Bush RT, Sullivan LA, Hocking RK, Mitchell DRG, Johnston SG, Fitzpatrick RW, Raven M, McClure S, Jang LY. 2009 Iron-monosulfide oxidation in natural sediments: Resolving microbially mediated S transformations using XANES, electron microscopy, and selective extractions. Environ Sci Technol 43:3128-3134. 